\title{
Сравнительный анализ инжекционных микродисковых лазеров на основе квантовых ям InGaAsN и квантовых точек InAs/InGaAs
}

\author{
(C) Э.И. Моисеев ${ }^{1}$, М.В. Максимов ${ }^{1}$, Н.В. Крыжановская ${ }^{1,2}$, О.И. Симчук ${ }^{1}$, М.М. Кулагина ${ }^{3}$, \\ C.A. Кадинская ${ }^{1}$, M. Guina ${ }^{4}$, A.E. Жуков ${ }^{1,2, \text { ฯ }}$ \\ ${ }^{1}$ Санкт-Петербургский национальный исследовательский Академический университет Российской академии наук, \\ 194021 Санкт-Петербург, Россия \\ ${ }^{2}$ Санкт-Петербургский политехнический университет Петра Великого, \\ 195251 Санкт-Петербург, Россия \\ ${ }^{3}$ Физико-технический институт им. А.Ф. Иоффе Российской академии наук, \\ 194021 Санкт-Петербург, Россия \\ ${ }^{4}$ Tampere University of Technology, \\ 33720 Tampere, Finland \\ "E-mail: zhukale@gmail.com
}

Поступила в Редакцию 20 октября 2019 г.

В окончательной редакции 29 октября 2019 г.

Принята к публикации 29 октября 2019 г.

\begin{abstract}
Представлены результаты сравнительного анализа спектральных и пороговых характеристик работающих при комнатной температуре инжекционных микродисковых лазеров спектрального диапазона 1.2xх мкм с разной активной областью: квантовые ямы InGaAsN/GaAs или квантовые точки InAs/InGaAs/GaAs. Обнаружено, что микролазеры сравнимого с квантовыми ямами размера обладают бо́льшими значениями порога лазерной генерации по сравнению с микролазерами с квантовыми точками. В то же время последние характеризуются заметно меньшей долей излучаемой мощности, приходящейся на лазерные моды. Также для них характерен перескок к генерации через возбужденный оптический переход. Этих недостатков лишены микродисковые лазеры на основе InGaAsN.
\end{abstract}

Ключевые слова: микролазер, квантовые ямы, квантовые точки, азотсодержащие полупроводники.

DOI: $10.21883 /$ FTP.2020.02.48907.9290

\section{1. Введение}

Микродисковые и микрокольцевые лазеры привлекают к себе широкое внимание в силу перспективности их использования в качестве сверхмалых источников излучения. Преимуществами таких лазеров являются простота изготовления, малый размер, низкие пороговые токи и направленность излучения в плоскости резонатора. Хотя первые микродисковые лазеры были изготовлены на основе InGaAs/InGaAsP/InP [1], в последние годы значительное внимание уделяется микролазерам на основе гетероструктур $\mathrm{AlGaAs} / \mathrm{GaAs}$ в сочетании с активной областью на основе квантовых точек (КT) In $(\mathrm{Ga}) \mathrm{As}$ [2]. Они также позволяют достичь генерации в телекоммуникационном спектральном диапазоне (вблизи 1.3 мкм) и при этом по сравнению с гетероструктурами в системе InGaAsP характеризуются более высокой температурной стабильностью благодаря большей глубине локализации носителей заряда и более высокой теплопроводностью [3]. Для таких микролазеров с КТ были получены низкие пороговые токи [4], генерация при температурах выше $100^{\circ} \mathrm{C}$ [5], показана возможность создания низкопороговых микролазеров, монолитно интегрированных с кремнием [6], в том числе работающих при повышенных температурах [7].

В настоящее время предполагается, что основной областью применения микродисковых лазеров будет опти- ческая передача данных на сверхмалые расстояния (между платами и в пределах платы между чипами) [8-10]. Вместе с тем, если удастся достичь достаточно высоких значений выходной мощности в лазерной моде шепчущей галереи и быстродействия $>10$ Гб/с, подобные микролазеры могут стать альтернативой сложным в изготовлении одночастотным лазерам с распределённой обратной связью и вертикально-излучающим лазерам для оптической передачи данных на средние и малые расстояния, работающим, например, во втором окне прозрачности оптического волокна вблизи 1.31 мкм. Так, в работе [11] сообщалось о передаче данных по одномодовому волокну со скоростью 7 Гб/с на расстояние 50 км с использованием в качестве источника сигнала микрокольцевых лазеров диаметром 40-80 мкм.

Необходимым требованием к лазерному источнику, предназначенному для использования в системах оптической связи, является достаточно высокая оптическая мощность, излучаемая на стабильной частоте. В то же время для микролазеров с КТ присущи перескоки мод с ростом тока инжекции [12], насыщение интенсивности моды с ростом накачки [13], одновременная генерация нескольких мод [14] и низкая интенсивность лазерных мод по отношению к спонтанному излучению [15], что, вероятно, связано с низкой плотностью электронных состояний в КТ и с неоднородным уширением оптических переходов. Этих недостатков могут быть лише- 
$a$

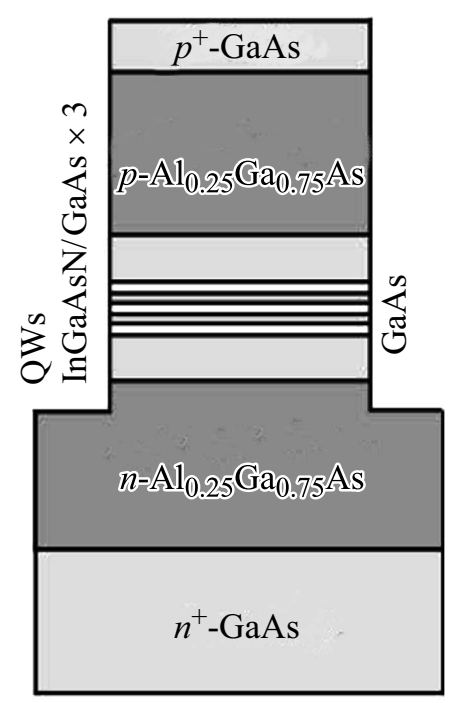

$b$

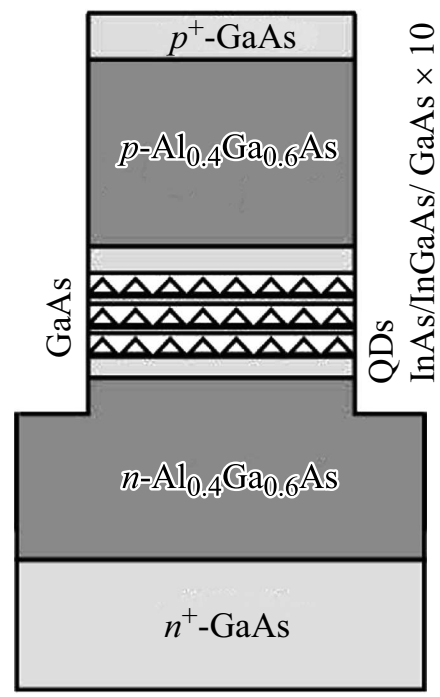

$c$

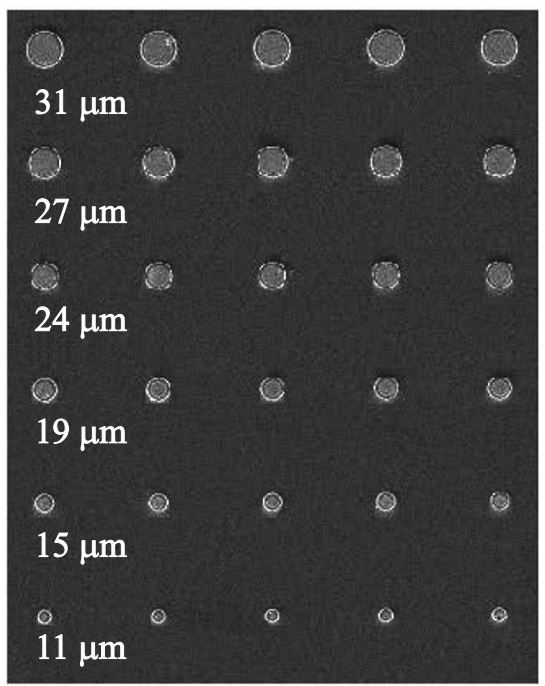

Рис. 1. Схематическое изображение последовательности слоев лазерных гетероструктур на основе азотсодержащих квантовых ям (QWs) $(a)$, квантовых точек (QDs) $(b)$ и фотография массива микродисковых лазеров с КЯ InGaAsN разного диаметра $(c)$.

ны микролазеры на квантовых ямах (КЯ). Фактически единственными КЯ на подложках GaAs, позволяющими реализовать длинноволновую лазерную генерацию, являются азотсодержащие КЯ $\operatorname{InGaAsN}(\mathrm{Sb})$ [16]. Недавно нами были впервые реализованы инжекционные микродисковые лазеры на основе КЯ InGaAsN, способные работать при комнатной температуре [17]. В дальнейшем характеристики таких микролазеров были улучшены за счет оптимизации эпитаксиальной гетероструктуры и изготовления микрорезонатора.

Целью настоящей работы является исследование спектральных и пороговых характеристик микродисковых лазеров с активной областью, представляющей собой двумерные КЯ InGaAsN/GaAs, и сопоставление со свойствами аналогичных микролазеров на основе КТ InAs/InGaAs.

\section{2. Особенности эксперимента}

Гетероструктуры, использованные для создания микродисковых лазеров, были синтезированы методом молекулярно-пучковой эпитаксии на подложках $n^{+}-\mathrm{GaAs}(100)$ (рис. 1, $a, b$ ). Для роста азотсодержащей лазерной структуры применялся источник с плазменной активацией азота. Активная область структура представляла собой 3 напряженные КЯ $\mathrm{Ga}_{0.7} \mathrm{In}_{0.3} \mathrm{~N}_{0.02} \mathrm{As}_{0.98}$, отделенные друг от друга прослойками GaAs толщиной 10 нм и помещенные в нелегированный волноводный слой GaAs толщиной 0.4 мкм. Эмиттерные слои $\mathrm{Al}_{0.25} \mathrm{Ga}_{0.75} \mathrm{As} n$ - и $p$-типа проволимости по обе стороны волновода имели толщину 2 мкм. Лазерная структура с квантовыми точками содержала такой же волновод, в котором были расположены 10 рядов КТ
InAs/InGaAs, отделенных друг от друга слоями GaAs толщиной 30 нм. Мольная доля арсенида алюминия в эмиттерных слоях толщиной 2.2 мкм составляла 25\%. Обе эпитаксиальные структуры заканчивались сильно легированным контактным слоем $p^{+}$-GaAs.

Микродисковые резонаторы были сформированы с помощью плазменного травления сквозь волноводный слой. Диаметр мез варьировался от $\sim 10$ до 30 мкм (рис. 1,c). Пассивация боковых граней не использовалась. Для создания сплошного омического контакта к подложке была применена металлизация $\mathrm{AuGe} / \mathrm{Ni} / \mathrm{Au}$. Индивидуальные верхние контакты имели круглую форму и были сформированы с помощью металлизации $\mathrm{AgMn} / \mathrm{NiAu}$. На верхний контакт устанавливался металлический зонд $\mathrm{W} / \mathrm{Rh}$, подложка располагалась на проводящей поверхности.

Микролазеры исследовались при импульсной накачке с длительностью импульсов 200 нс и частотой повторения 100 кГц. Измерения были выполнены при комнатной температуре без принудительного охлаждения. Сбор излучения проводился с помощью микрообъектива Mitutoyo M Plan Apo NIR 50X с числовой апертурой 0.42 , обеспечивающей угол сбора около $50^{\circ}$. Собранное излучение вводилось в оптоволокно через адаптер и затем поступало в оптический анализатор спектров Yokogawa AQ6370C, работающий в диапазоне длин волн 600-1700 нм и имеющий встроенный калибратор измеряемой мощности сигнала. Если не указано иное, спектры записывались с разрешением 200 пм. Доля интенсивности, приходящейся на лазерные моды, определялась по отношению к полной спектральной интенсивности сигнала электролюминесценции, измеряемой в диапазоне длин волн 950-1350 нм. 

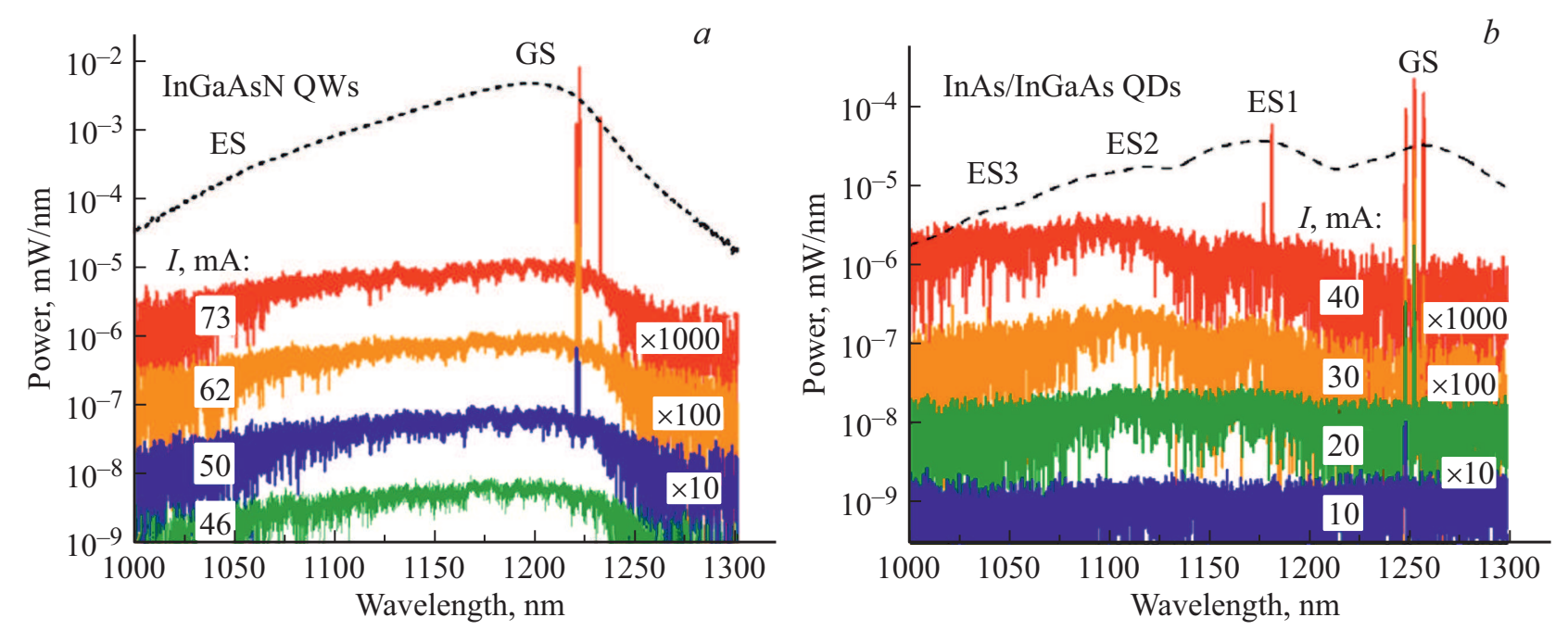

Рис. 2. Спектры излучения микродисков диаметром 31 мкм с КЯ $\operatorname{InGaAsN}(a)$ и КТ InAs/InGaAs $(b)$ при разных токах инжекции $I$. Штриховая линия: спектр излучения планарной структуры при $700 \mathrm{~A} / \mathrm{cm}^{2}$.

\section{3. Экспериментальные результаты и обсуждение}

На рис. 2 штриховыми линиями представлены измеренные при низком уровне возбуждения $\left(\sim 700 \mathrm{~A} / \mathrm{cm}^{2}\right)$ и с низким спектральным разрешением (1 нм) спектры излучения, записанные с таких участков лазерных гетероструктур, на которых не происходило формирование мез. Таким образом, эти спектры излучения позволяют судить о длине волны оптических переходов соответствующей активной области: квантовые ямы (рис. 2, $a$ ) и квантовые точки (рис. 2,b). В случае КЯ InGaAsN основной оптический переход (GS) имеет максимум на длине волны 1.2 мкм. Возбужденный переход (ES), наблюдаемый в виде слабо выраженного коротковолнового плеча, заметно отстоит от основного перехода и лежит вблизи 1.06 мкм. Структура с KT InAs/InGaAs характеризуется меньшим спектральным разделением между основным (GS, 1.26 мкм) и первым возбужденным (ES1, 1.18 мкм) переходами. В спектре излучения КТ также наблюдаются более коротковолновые возбужденные оптические переходы высших порядков: ES2 (1.11 мкм) и $\mathrm{ES} 3$ (1.04 мкм).

На рис. 2 также показаны снятые при различных токах накачки спектры излучения микродисковых лазеров диаметром 31 мкм с двумя типами активной области. Для удобства восприятия спектры сдвинуты по вертикальной оси. В микродиске с КЯ InGaAsN дублет узких линий излучения (1219.72 и 1221.16 нм), соответствующих модам шепчущей галереи, возникает по достижении порога лазерной генерации на длинноволновом крае пика спонтанного излучения. Пороговый ток оценен равным 50 мА. Это соответствует пороговой плотности тока $6.6 \kappa \mathrm{A} / \mathrm{cm}^{2}$, что в 1.5 раза меньше, чем для ранее представленных микродисковых лазерах такого же размера с квантовыми ямами InGaAsN [17]. По мере уве-

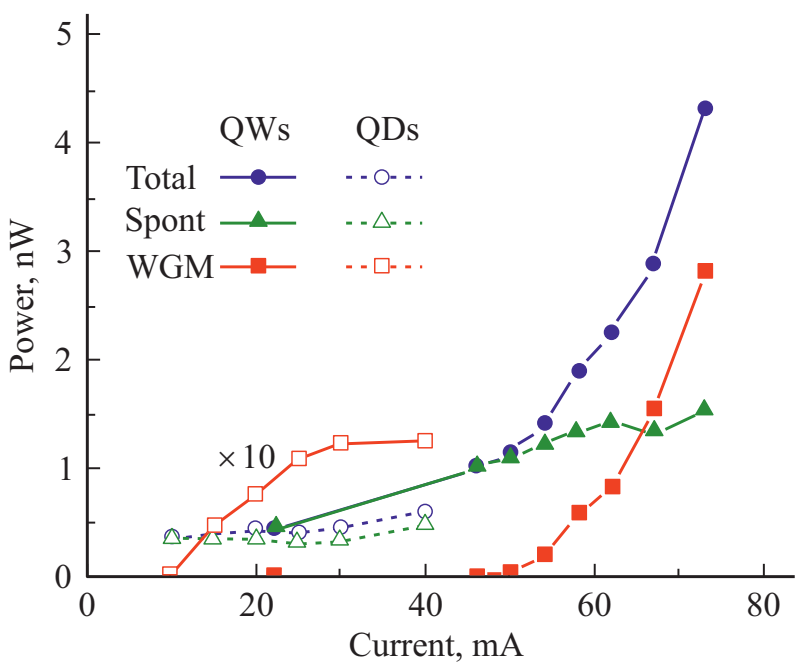

Рис. 3. Зависимость интенсивности излучения микродисков диаметром 31 мкм с КЯ (QWs) и КТ (QDs): полная интенсивность (Total), спонтанное излучение (Spont), лазерные моды шепчущей галереи (WGM).

личения тока накачки выше порога вплоть до 70 мА не наблюдается длинноволнового сдвига положения моды шепчущей галереи, что свидетельствует об отсутствии разогрева активной области, однако в спектре возникает дополнительная более длинноволновая лазерная мода (1231.64 нм). При этом интенсивность спонтанного излучения с ростом тока насыщается, тогда как интегральная интенсивность излучения мод шепчущей галереи быстро возрастет (рис. 3). В результате доля лазерного излучения в общей излучаемой микролазером оптической мощности растет и при наибольшем использованном токе инжекции превышает $60 \%$.

Рис. 4, $a$ обобщает полученные в микродисковых лазеpax на основе КЯ InGaAsN зависимости доли лазерного 

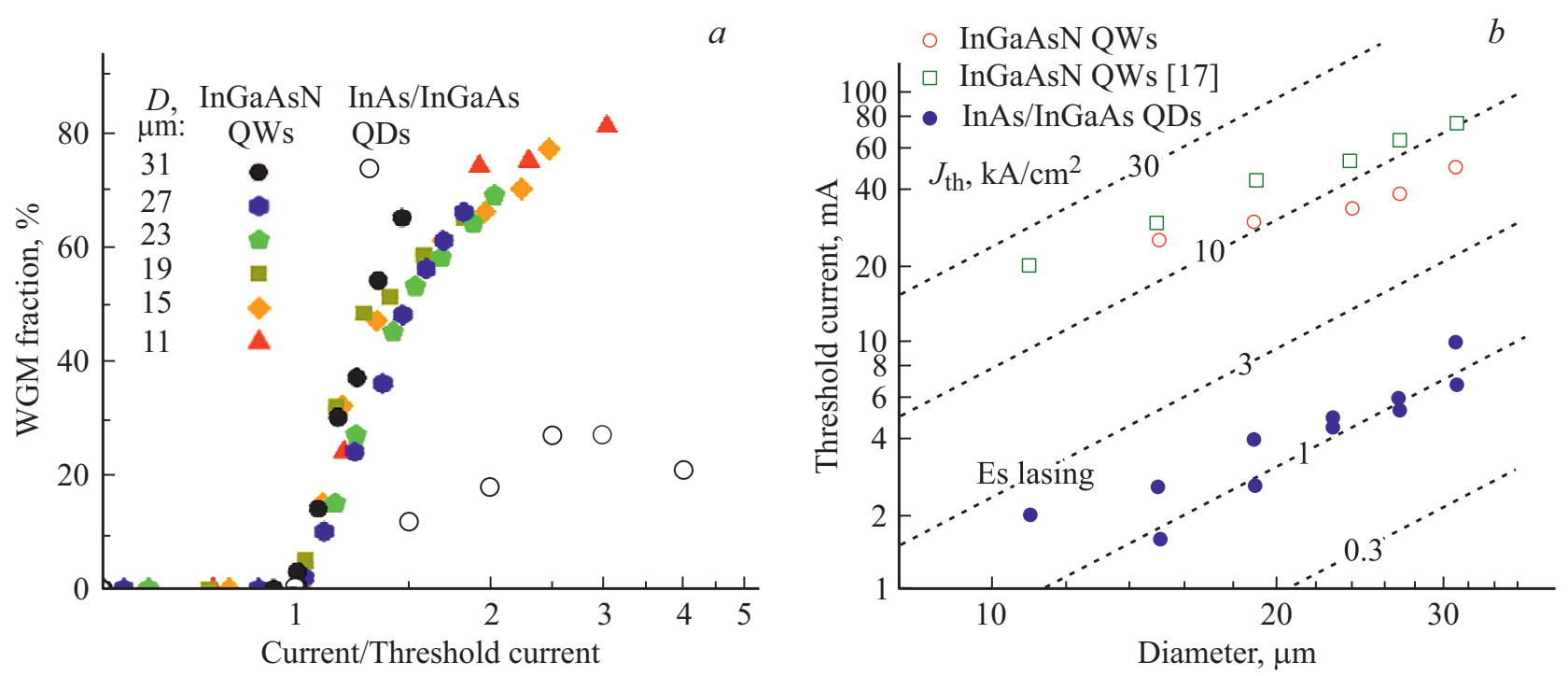

Рис. 4. Доля лазерных мод в полной интенсивности излучения $(a)$ и пороговый ток $(b)$ для микролазеров разного диаметра $D$ на основе КЯ (QWs) и KT (QDs). Штриховые линии соответствуют фиксированным значениям плотности тока $J_{\text {th }}$.

излучения от тока инжекции. Для удобства сравнения величина тока нормирована на значение порогового тока, определенного для данного образца. Как видно, в этих координатах зависимости, полученные для микролазеров разного диаметра, практически совпадают. Наибольшее значение доли лазерного излучения в полной оптической мощности микролазера составило $81 \%$ и было измерено в микродиске диаметром 11 мкм при токе инжекции 41 мА (трехкратное превышение порога генерации).

Спектральные характеристики микролазеров с активной областью в виде массива КТ заметно отличаются (рис. 2, b). Наблюдаемая при относительно низких токах $(\leqslant 30$ мА $)$ группа мод расположена в пределах полосы основного оптического перехода КТ. При этом спектральное положение лазерных мод (1249.08, 1253.32 и 1258.0 нм) близко к максимуму спонтанного излучения основного перехода, а не сдвинуто в длинноволновую область, как это наблюдалось для микродисков с КЯ. В результате, хотя длины волн основных переходов структур с КТ и КЯ отличаются друг от друга заметно (1.26 и 1.2 мкм соответственно), лазерная генерация в этих структурах оказывается в близких спектральных диапазонах ( 1.25 и 1.22 мкм).

Кроме того, микролазеры на основе КТ характеризуются заметно меньшей долей лазерного излучения в полной излучаемой мощности (рис. $4, a$ ). Максимальное измеренное значение составило $27 \%$ при накачке, равной 2.5-3 пороговых тока, после чего интенсивность лазерных линий насыщается (рис. 3), а доля лазерного излучения спадает (рис. 4,a). Это сопровождается возникновением при высоких токах инжекции $(\geqslant 40 \mathrm{MA})$ дополнительной группы мод (1177.52 и 1181.76 нм), спектральное положение которых соответствует первому возбужденному оптическому переходу. Подобное поведение, получившее название двухчастотная генерация, ранее наблюдалось в лазерах полосковой конструкции с КТ и было объяснено медленным захватом носителей заряда на основной квантовый уровень КТ в сочетании с большей степенью вырождения возбужденного уровня $[18,19]$.

Микродисковые лазеры с КТ имеют по сравнению с микродисками на основе КЯ меньший порог лазерной генерации. На рис. 4, $b$ приведены экспериментальные данные, полученные нами как в настоящей, так и в более ранних работах. Наклонные штриховые линии позволяют оценить пороговую плотность тока. В целом микродисковые лазеры с КТ характеризуются плотностью порогового тока $\sim 1 \mathrm{\kappa A} / \mathrm{cm}^{2}$. Пороговая плотность тока в исследованных микродисковых лазерах с КЯ InGaAsN возрастает при уменьшении диаметра с 6.6 до 14 кА/ $\mathrm{cm}^{2}$, что может быть связано с влиянием безызлучательной рекомбинации на боковых стенках микрорезонатора. Мы полагаем, что снижения порога генерации в микродисках на основе КЯ можно достичь за счет применения пассивации боковых стенок [20]. Также следует отметить, что лазерная генерация в микролазере с КТ наименьшего диаметра сразу начиналась на длине волны, отвечающей первому возбужденному переходу, тогда как генерация всех микродисков с КЯ происходит через основной оптический переход в КЯ.

\section{4. Заключение}

Таким образом, нами были исследованы работающие при комнатной температуре инжекционные микродисковые лазеры диаметром 11-31 мкм с активной областью в виде квантовых ям InGaAsN/GaAs с длиной волны генерации > 1.2 мкм. Длина волны генерации во всем диапазоне исследованных диаметров микрорезонаторов и при разных токах накачки лежит в спектральном 
диапазоне основного оптического перехода, минимальное значение пороговой плотности тока составило 6.6 кА/см². Микролазеры на основе азотсодержащих КЯ характеризуются отсутствием при больших токах насыщения интенсивности лазерной мощности, излучаемой через моды шепчущей галереи, и ее высокой долей $(65-81 \%)$ в общей оптической мощности, излучаемой в свободное пространство. Полученные спектральные характеристики способствуют использованию микролазеров на основе квантовых ям InGaAsN для передачи данных в системах оптической связи.

\section{Финансирование работы}

Работа выполнена при поддержке гранта РФФИ 1629-03127 офи_м и Министерства образования и науки России (3.9787.2017/8.9).

\section{Конфликт интересов}

Авторы заявляют, что у них нет конфликта интересов.

\section{Список литературы}

[1] A.F.J. Levi, R.E. Slusher, S.L. McCall, T. Tanbuk-Ek, D.L. Coblentz, S.J. Perton. Electron. Lett., 28, 1010 (1992).

[2] L. Zhang. E. Hu. Appl. Phys. Lett., 82, 319 (2003).

[3] A.K. Sokol, R.P. Sarzala. Opt. Appl., 43, 325 (2013).

[4] M.-H. Mao, H.-C. Chien, J.-Z. Hong, C.-Y. Cheng. Opt. Express, 19, 14145 (2011).

[5] E. Moiseev, N. Kryzhanovskaya, M. Maximov, F. Zubov, A. Nadtochiy, M. Kulagina, Y. Zadiranov, N. Kalyuzhnyy, S. Mintairov, A. Zhukov. Optics. Lett., 43, 4554 (2018).

[6] Y. Wan, J. Norman, Q. Li, M.J. Kennedy, D. Liang, C. Zang, D. Huang, Z. Zhang, A.Y. Liu, A. Torres, D. Jung, A.C. Gossard, E.L. Hu, K.M. Lau, J.E. Bowers. Optica, 4, 940 (2017).

[7] N.V. Kryzhanovskaya, E.I. Moiseev, Y.S. Polubavkina, M.V. Maximov, D.V. Mokhov, I.A. Morozov, M.M. Kulagina, Y.M. Zadiranov, A.A. Lipovskii, M. Tang, M. Liao, J. Wu, S. Chen, H. Liu, A.E. Zhukov. Laser Phys. Lett., 15, 015802 (2018).

[8] Y.-D. Yang, Y. Zhang, Y.-Z. Huang, A.W. Poon. Opt. Express, 22, 824 (2014).

[9] Y. Wan, D. Inoue, D. Jung, J.C. Norman, C. Shang, A.C. Gossard, J.E. Bowers. Photon. Res., 6, 776 (2018).

[10] N.V. Kryzhanovskaya, E.I. Moiseev, F.I. Zubov, A.M. Mozharov, M.V. Maximov, N.A. Kalyuzhnyy, S.A. Mintairov, M.M. Kulagina, S.A. Blokhin, K.E. Kudryavtsev, A.N. Yablonskiy, S.V. Morozov, Yu. Berdnikov, S. Rouvimov, A.E. Zhukov. Photon. Res., 7, 664 (2019).

[11] A. Kapsalis, D. Syvridis, U. Troppenz, M. Hamacher, H. Heidrich. Proc. Conf. Optical Fiber Communication/National Fiber Optic Engineers Conf. (2008) p. 2917.

[12] N.V. Kryzhanovskaya, E.I. Moiseev, Yu.V. Kudashova, F.I. Zubov, A.A. Lipovskii, M.M. Kulagina, S.I. Troshkov, Yu.M. Zadiranov, D.A. Livshits, M.V. Maximov, A.E. Zhukov. Electron. Lett., 51, 1354 (2015).

[13] T. Ide, T. Baba, J. Tatebayashi, S. Iwamoto, T. Nakaoka, Y. Arakawa. Appl. Phys. Lett., 85, 1326 (2004).
[14] Y. Wan, Q. Li, A.Y. Liu, A.C. Gossard, J.E. Bowers, E.L. Hu, K.M. Lau. Appl. Phys. Lett., 109, 011104 (2016).

[15] Ф.И. Зубов, Н.В. Крыжановская, Э.И. Моисеев, Ю.С. Полубавкина, О.И. Симчук, М.М. Кулагина, Ю.М. Задиранов, С.И. Трошков, А.А. Липовский, М.В. Максимов, А.Е. Жуков. ФТП, 50, 1425 (2016).

[16] K. Nakahara, M. Kondow, T. Kitatani, M.C. Larson, K. Uomi. IEEE Photon. Technol. Lett., 10, 487 (1998).

[17] E.I. Moiseev, M.V. Maximov, A.M. Nadtochiy, N.V. Kryzhanovskaya, D.A. Sannikov, T. Yagafarov, M. Kulagina, T. Niemi, R. Isoaho, M. Guina, A.E. Zhukov. IOP J. Phys.: Conf. Ser., 1124, 081048 (2018).

[18] E.A. Viktorov, P. Mandel, Y. Tanguy, J. Houlihan, G. Huyet. Appl. Phys. Lett., 87, 053113 (2005).

[19] V.V. Korenev, A.V. Savelyev, A.E. Zhukov, A.V. Omelchenko, M.V. Maximov. Appl. Phys. Lett., 102, 112101 (2013).

[20] N.V. Kryzhanovskaya, M.V. Lebedev, T.V. Lvova, Yu.V. Kudashova, I.I. Shostak, E.I. Moiseev, A.E. Zhukov, M.V. Maximov, M.M. Kulagina, A.M. Nadtochiy, S.I. Troshkov. IOP J. Phys.: Conf. Ser., 643, 012043 (2015).

Редактор Л.В. Шаронова

\section{Comparative analysis of diode microdisk lasers based of InGaAsN quantum wells and InAs/InGaAs quantum dots}

E.I. Moiseev ${ }^{1}$, M.V. Maximov ${ }^{1}$, N.V. Kryzhanovskaya ${ }^{1,2}$, O.I. Simchuk ${ }^{1}$, M.M. Kulagina ${ }^{3}$, S.A. Kadinskaya ${ }^{1}$, M. Guina ${ }^{4}$, A.E. Zhukov ${ }^{1,2}$

${ }^{1}$ St. Petersburg National Research

Academic University,

Russian Academy of Sciences,

194021 St. Petersburg, Russia

2 Peter the Great Polytechnic University,

195251 St. Petersburg, Russia

${ }^{3}$ loffe Institute,

194021 St. Petersburg, Russia

${ }^{4}$ Tampere University of Technology,

33720 Tampere, Finland

Abstract The results are presented on a comparative analysis of spectral and threshold characteristics of diode microdisk lasers operating at room temperature in a spectral range of $1.2 \mathrm{xx} \mu \mathrm{m}$ with different active regions: $\mathrm{InGaAsN} / \mathrm{GaAs}$ quantum wells or InAs/InGaAs/GaAs quantum dots. It was found that microlasers of a comparable size with quantum wells have higher lasing threshold compared to microlasers with quantum dots. At the same time, the latter are characterized by a noticeably smaller fraction of the radiated power with the laser modes. They are also characterized by a jump to excited-state optical transition lasing. The InGaAsNbased microdisk lasers lack these disadvantages. 